\title{
Light axigluon and single top production at the $\mathrm{LHC}$
}

\author{
Chong-Xing Yue, Shi-Yue Cao and Qing-Guo Zeng \\ Department of Physics, Liaoning Normal University, \\ Dalian 116029, P.R. China \\ E-mail: cxyue@lnnu.edu.cn, 799514165@qq.com, zengqingguo66@126.com
}

ABSTRACT: The light axigluon model can explain the Tevatron $t \bar{t}$ forward-backward asymmetry and at the same time satisfy the constraints from the electroweak precision measurement and the $A T L A S$ and $C M S$ data, which induces the flavor changing $(F C)$ couplings of axigluon with the $S M$ and new quarks. We investigate the effects of these $F C$ couplings on the s- and t-channel single top productions at the $L H C$ and the $F C$ decays $Z \rightarrow \bar{b} s+b \bar{s}$, $t \rightarrow c \gamma$ and $c g$. Our numerical results show that the light axigluon can give significantly contributions to single top production and the rare top decays $t \rightarrow c \gamma$ and $c g$.

Keywords: Phenomenological Models, Hadronic Colliders

ARXIV EPRINT: 1401.5159 


\section{Contents}

1 Introduction 1

2 Light axigluon and the $F C$ decays $Z \rightarrow \bar{b} s$ and $b \bar{s} \quad 2$

3 The $F C$ couplings of the light axigluon $A$ and single top production at the $\mathrm{LHC}$

4 The light axigluon and the rare top decays $t \rightarrow c \gamma$ and $c g$

5 Conclusions

\section{Introduction}

The standard model $(S M)$ of particle physics has been proven to be extremely successful describing collider experimented data so far. Even the discovery of a Higgs-like particle [1, 2] has confirmed the validity of the $S M$ at the Fermi scale. However, the $S M$ suffers from a key theoretical drawback, the so-called "hierarchy" problem, which means that it could be a low-energy effective theory valid only up to some cut-off energy scale $\Lambda$, about $\mathrm{TeV}$ scale. So new physics beyond the $S M$ would be in an energy range accessible at the $L H C$ and might be discovered in coming years, although, at the moment, there is not any collider hint of new physics at the $L H C$.

There are various new physics models extending the gauge group of the strong interaction sector give rise to massive color-octet vector boson, for example, the topcolor models [3-6] and chiral color models [7-12]. Other examples include the extra dimensional models [13] and technicolor [14, 15], which predict the existence of the Kaluza-Klein (KK) gluons and technirhos, respectively. Among these color-octet vector bosons, the new paricles with axial-vector couplings to the $S M$ quarks are called "axigluons", which might explain the anomalous forward-backward asymmetry $(F B A)$ in the $t \bar{t}$ production observed at the Tevatron [16-18]. So far, there has been a significant amount of works to explain the $t \bar{t} F B A$ via axigluons, for example see [19-40]. Furthermore, the light axigluon $A$ with a mass $M_{A}$ in the range from $100 \mathrm{GeV}$ to $400 \mathrm{GeV}$ can explain the $t \bar{t} F B A$ and satisfy the constraints from the $A T L A S$ and $C M S$ data [41-50], as long as its decay width is large and its couplings to the $S M$ quarks are relatively small [33-39].

Top quark physics is expected to be a window to any new physics beyond the electroweak scale. At $L H C$ energies, top quark is copiously produced both in pair and single productions, which allows for an unprecedented precision in the study of top observables, such as its couplings and rare decays [51-53]. At hadron colliders, single top quark production is an important process in probing the mechanism of electroweak symmetry breaking 
( $E W S B$ ), providing informations complementary to those that can be obtained from top pair production [54-56]. Single top production is also very sensitive to new physics effects, whose strength can be assessed by precise measurement of the production cross section.

Single top production at hadron colliders has been observed in three channels: schannel, t-channel [57-66] and $t W$ associated production channel [67, 68], which accord with the $S M$ predictions within experimental uncertainties. ATLAS and CMS collaborations have started searching for the new physics effects on single top production.

Inspired by the solution of the light axigluon to the $t \bar{t} F B A$, some axigluon-mediated phenomena are studied in this paper. We consider the contributions of the light axigluon with flavor changing $(F C)$ couplings to the $S M$ and new quarks to the $F C$ decays $Z \rightarrow$ $\bar{b} s(b \bar{s})$, the s- and t-channel single top productions, and rare top decays $t \rightarrow c \gamma$ and $c g$ in the context of the light axigluon model proposed by Tavares and Schmaltz [36, 37]. The constraints on this new physics model from the electroweak precision observables and the relevant data given by hadron colliders are taken into account in our numerical calculations.

The rest of this paper is organized as follows: After reviewing the basic ingredients of the light axigluon model, in section 2, we calculate the contributions of the light axigluon to the $F C$ decays $Z \rightarrow \bar{b} s$ and $b \bar{s}$. Corrections of the light axigluon to the cross sections of the s- and t-channel single top productions at the $L H C$ are studied in section 3 . The branching ratios of the rare top decays $t \rightarrow c \gamma$ and $c g$ induced by light axigluon exchange are given in section 4 . Section 5 is devoted to simple summary.

\section{Light axigluon and the $F C$ decays $Z \rightarrow \bar{b} s$ and $b \bar{s}$}

The light axigluon model $[36,37]$ is based on the gauge group $G=\mathrm{SU}(3)_{1} \times \mathrm{SU}(3)_{2} \times \mathrm{SU}(2) \times$ $\mathrm{U}(1)_{Y}$, where $\mathrm{SU}(2) \times \mathrm{U}(1)_{Y}$ is the conventional electroweak group and the extended gauge group $\mathrm{SU}(3)_{1} \times \mathrm{SU}(3)_{2}$ is spontaneously broken to the $Q C D$ gauge group $\mathrm{SU}(3)_{C}$ by the vacuum expectation value $(V E V)$ of a bifundamental scalar $\phi$. This breaking pattern yields two mass eigenstates of color-octet gauge bosons. One is massless particle, which can be identified with the $S M$ gluon, and the other is massive particle, which is called the light axigluon $A$. For its couplings to the $S M$ quarks, there are the vector coupling $g_{V} \approx 0$ and the axial-vector coupling $g_{A} \neq 0$ in the case of assuming approximately parity symmetry. In order to cancel the gauge anomaly, the extra up- and down-type quarks are introduced into this model, and the lepton sector is exactly same as that of the $S M$. To explain the $t \bar{t} F B A$, the axigluon $A$ should have mass below $450 \mathrm{GeV}$, while should be broad with $\Gamma_{A} / M_{A} \sim 10 \sim 20 \%$, where $\Gamma_{A}$ and $M_{A}$ represent its total decay width and mass, respectively.

In the original light axigluon model $[36,37]$, the authors assume the existence of an exact global symmetry of the axigluon couplings, and thus the light axigluon only has flavor universal couplings to the $S M$ quarks. In fact, this global symmetry is only approximate and there is mixing between new and ordinary quarks, which can induce flavor changing neutral currents (FCNCs) at tree level [69]. The new and ordinary quarks have same $\mathrm{SU}(2) \times \mathrm{U}(1)$ charge, their mixing does not give rise to the $F C Z$ couplings at tree level. 
The new scalars can not induce $F C N C s$, thus the non-universal axigluon couplings are the main source of $F C N C$ for this model.

In this paper we will not assume the existence of an exact global symmetry of the axigluon couplings, which allows $F C$ couplings of the axigluons to the $S M$ quarks. If one assumes that these $F C$ couplings are only axial-vector couplings, which are similar with their flavor conserving couplings to the $S M$ quarks, then the axial-vector couplings of the light axigluon to the $S M$ quarks can be general given by the Lagrangian

$$
\mathcal{L} \supset g_{s}\left[\bar{u}_{i} \gamma_{\mu} \gamma_{5}\left(g_{A}^{u_{i}} \delta_{i j}+\varepsilon_{u}^{i j}\right) u_{j} A^{\mu}+\bar{d}_{i} \gamma_{\mu} \gamma_{5}\left(\left(g_{A}^{d_{i}} \delta_{i j}+\varepsilon_{d}^{i j}\right) d_{j} A^{\mu}\right]\right.
$$

where $A^{\mu}$ is the light axigluon, $g_{s}$ is the $Q C D$ coupling constant, $u_{i}$ and $d_{i}$ are the $S M$ up- and down-type quarks, respectively. In above equation, we have neglected the color and spinor indices. $g_{A}^{u_{i}}$ and $g_{A}^{d_{i}}$ are the flavor independent coupling constants and there are $g_{A}^{u_{i}}=g_{A}^{d_{i}}=g_{A}^{q}[36,37]$. The $F C$ coupling constants $\varepsilon_{u}^{i j}$ and $\varepsilon_{d}^{i j}$, which arise from flavor symmetry breaking of new and light quarks, are given by the matrices

$$
\varepsilon_{u}=\left(\begin{array}{ccc}
0 & g^{u c} & g^{u t} \\
\left(g^{u c}\right)^{*} & 0 & g^{c t} \\
\left(g^{u t}\right)^{*} & \left(g^{c t}\right)^{*} & 0
\end{array}\right), \quad \varepsilon_{d}=\left(\begin{array}{ccc}
0 & g^{d s} & g^{d b} \\
\left(g^{d s}\right)^{*} & 0 & g^{b s} \\
\left(g^{d b}\right)^{*} & \left(g^{b s}\right)^{*} & 0
\end{array}\right) .
$$

The couplings of the axigluon to a pair of ordinary quarks and to the corresponding partners have opposite sign. So, in order to get suppressed couplings of the ordinary quarks to the axigluon, the extra quarks and the $S M$ quarks should have mixing [36, 37, 39, 70, 71]. The mixing can be obtained by adding a Yukawa coupling involving a scalar field $\phi$ in addition to the quark field of $Q^{\prime}$ with $Q$. After the spontaneous breakdown of $\mathrm{SU}(3)_{1} \times$ $\mathrm{SU}(3)_{2} \rightarrow \mathrm{SU}(3)_{C}$ induced by the $V E V$ for $\phi$, the new quarks from the line combinations of $Q^{\prime}$ and $Q$ get masses, while their orthogonal combinations correspond to the $S M$ quarks remain massless, which get masses from the $S M$ Higgs $V E V$ via Yukawa couplings. In the mass eigenstates, the mixing couplings of the axigluon to ordinary and new quarks, which are assumed to be axial-vector couplings, can be general written as

$$
\mathcal{L}^{\prime} \supset g_{s} g_{A}^{m i x}\left[\bar{U}_{H i} \gamma_{\mu} \gamma_{5}\left(\varepsilon_{H u}^{i j}\right) u_{j} A^{\mu}+\bar{D}_{H i} \gamma_{\mu} \gamma_{5}\left(\varepsilon_{H d}^{i j}\right) d_{j} A^{\mu}\right] .
$$

$U_{H i}$ and $D_{H i}$ represent the up-type and down-type new quarks, respectively. For the mixing coupling constant $g_{A}^{m i x}$, there is the relation $\left(g_{A}^{m i x}\right)^{2}+\left(g_{A}^{q}\right)^{2}=1$. For the two matrices $\varepsilon_{H u}$ and $\varepsilon_{H d}$, they are related through the $S M C K M$ matrix: $\varepsilon_{H u}^{+} \varepsilon_{H d}=V_{C K M}$, which is similar with the case for the mixing between the T-odd and T-even quarks in the LHT model [72]. In this paper, we assume that both $\varepsilon_{H u}$ and $\varepsilon_{H d}$ are nearly equal to the identity matrix, which provides us with a set of minimal flavor mixing scenarios. We take as examples two simple cases:

Case I $\varepsilon_{H u}=I, \quad \varepsilon_{H d}=V_{C K M}$,

Case II $\varepsilon_{H d}=I, \quad \varepsilon_{H u}=V_{C K M}$.

In case I, the mixing coupling $g_{A}^{Q q}$ has no contributions to $D^{0}-\overline{D^{0}}$ mixing, while contributes to $B_{q}^{0}-\overline{B_{q}^{0}}$ and $K^{0}-\overline{K^{0}}$ mixings. For case II, it is obvious that the mixing coupling $g_{A}^{Q q}$ can only contribute to $D^{0}-\overline{D^{0}}$ mixing. Reference [69] has obtained the 


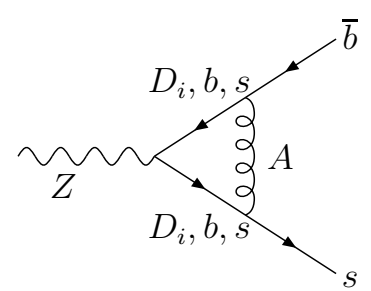

(a)

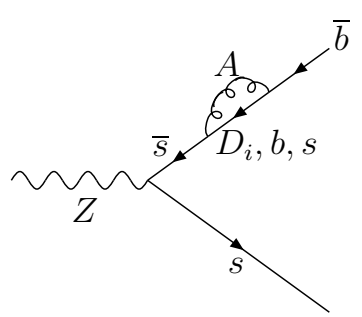

(b)

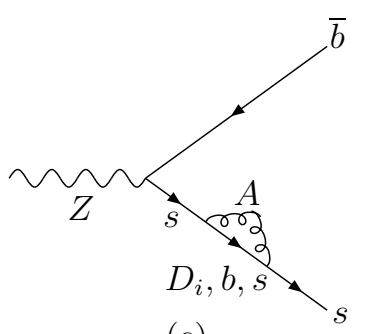

(c)

Figure 1. One-loop Feynman diagrams for the $F C$ decay $Z \rightarrow \bar{b} s$ induced by light axigluon exchange.

constraints on the mixing matrix $\varepsilon_{d}$ by using the available data from neutral meson mixings, such as $B_{q}^{0}-\overline{B_{q}^{0}}, K^{0}-\overline{K^{0}}$ and $D^{0}-\overline{D^{0}}$ mixings. Taking into account of these constants, in this section, we calculate the branching ratios of the $F C$ decays $Z \rightarrow \bar{b} s$ and $b \bar{s}$ given by axigluon exchange as shown in figure 1 . The self-energy diagrams figure $1(\mathrm{~b})$ and (c) contribute a finite field renormalization and the individual diagrams are finite [73]. To fulfill the broad width of the axigluon, the first and second generation new quarks should be degenerate and lighter than the axigluon, while the third generation new quarks must be heavier [36, 37]. So we think that the contributions of the third generation new quarks to the $F C$ decays $Z \rightarrow \bar{b} s(b \bar{s})$ decouple and only consider the contributions of the first and second generation new quarks. In our numerical estimation, we will take $M_{D_{H 1}}=M_{D_{H 2}}=M_{H}=0.2 M_{A}$. In this case, one can safely neglect the phase space suppression effect for the axigluon decaying to one new quark and one ordinary quark and there should be $\Gamma_{A} / M_{A} \sim 10 \sim 20 \%$.

The light axigluon model predicts the existence of new scalar, which also has the mixing couplings to new and ordinary quarks. However, it can not induce $F C$ couplings at tree level and thus in this paper we neglect the effects of the new scalar on the $F C$ processes $Z \rightarrow \bar{b} s$ and $b \bar{s}$.

The corrections of color-octet gauge boson to the $Z b \bar{b}$ coupling are firstly studied by ref. [74] in the context of topcolor models, which contain only the leading-logarithmic contributions. The full one-loop results for the corrections of the axigluon to the $Z b \bar{b}$ coupling are given in refs. [38, 39] in the case of neglecting the bottom quark mass. Ref. [39] have further computed the contributions from new quarks and new scalar to the $Z b \bar{b}$ coupling and find that the two kinds of contributions have opposite sign and the effect of new scalar is much smaller than that of new quarks. Following refs. [38, 39], we can straightforwardly calculate the contributions of the light axigluon model to the $F C$ couplings $Z \bar{b} s$ and $Z b \bar{s}$. Then, the effective $Z \bar{b} s$ coupling can be written as

$$
g_{P}^{Z b s}=\frac{\alpha_{s}}{3 \pi} g_{P}^{Z b b}\left[2 g_{P}^{A b b} g_{P}^{A b s} \kappa\left(x_{z}\right)+\left(g_{A}^{m i x}\right)^{2} \kappa\left(x_{z}, x_{h}\right)\left(\varepsilon_{H d}^{* 13} \varepsilon_{H d}^{12}+\varepsilon_{H d}^{* 23} \varepsilon_{H d}^{22}\right)\right],
$$

where $P=L$ and $R \cdot g_{P}^{Z b b}$ and $g_{P}^{A b b}$ represent the couplings of the gauge boson $Z$ and axigluon $A$ to the bottom quark pairs, respectively. The explicit expressions of the factors $\kappa\left(x_{Z}\right)$ and $\kappa\left(x_{z}, x_{h}\right)$ have been given in ref. [39]. Since the couplings of the axigluon to pair of ordinary quarks and pair of new quarks are flavor universal and the new and ordinary 


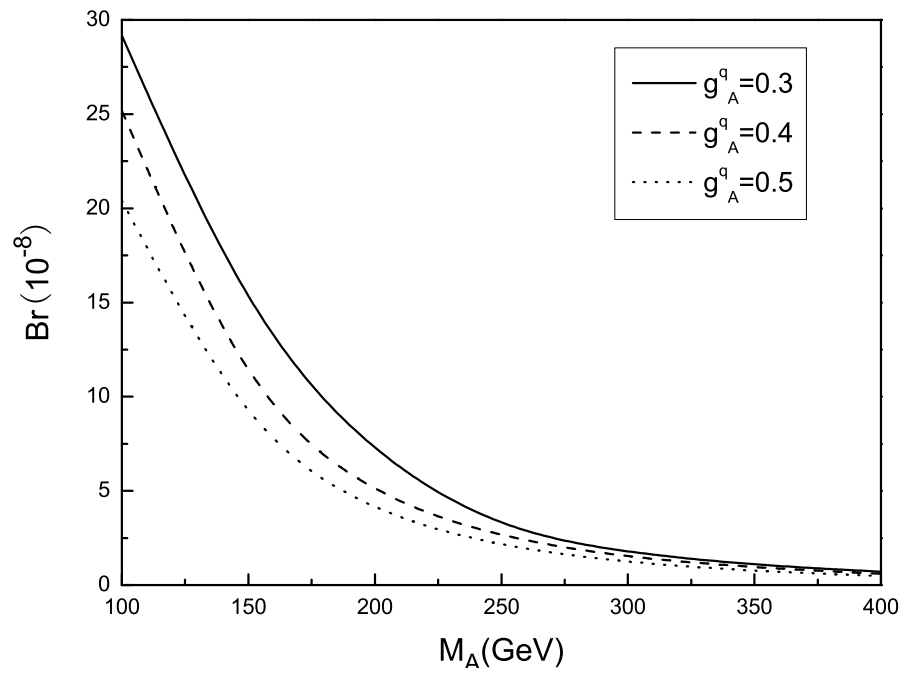

Figure 2. Variation of the branching ratio $\operatorname{Br}(Z \rightarrow \bar{b} s+b \bar{s})$ with the axigluon mass $M_{A}$ for $g_{A}^{b s}=1.83 \times 10^{-3}, \varepsilon_{H d}=V_{C K M}$ and three values of the coupling parameter $g_{A}^{q}$.

quarks have same $\mathrm{SU}(2) \times \mathrm{U}(1)$ charge, in above equation we have added the contributions of the ordinary quarks $b$ and $s$, and taken

$$
g_{L}^{Z b b}=g_{L}^{Z D_{i} D_{i}}=\frac{e}{4 S_{W} C_{W}}\left(1-\frac{2}{3} S_{W}^{2}\right), \quad g_{R}^{Z b b}=g_{R}^{Z D_{i} D_{i}}=-\frac{e}{4 S_{W} C_{W}} \cdot \frac{2}{3} S_{W}^{2},
$$

where $i=1$ and $2, S_{W}=\sin \theta_{W}$ and $C_{W}=\cos \theta_{W}, \theta_{W}$ is the Weinberg angle. The $F C$ coupling $g_{P}^{A b s}$ can contribute to $B_{s}^{0}-\overline{B_{s}^{0}}$ mixing at tree level and its upper bound has been obtained by ref. [69] as $\left|g_{L}^{b s}\right|=\left|g_{R}^{b s}\right|=\left|g_{A}^{b s}\right| \leq 1.83 \times 10^{-3}$. In fact, for the case I, the new quarks can also generate contributions to $B_{s}^{0}-\overline{B_{s}^{0}}$ mixing via box diagrams that contain the light axigluon and new quark. However, the contributions from box diagrams are suppressed with respect to axigluon tree-level contributions by a loop factor $1 /\left(16 \pi^{2}\right)$ and two additional mixing matrix elements $\varepsilon_{H d}^{i 3}$ and $\varepsilon_{H d}^{i 2}$. Therefore they cannot compete with the latter and are negligible. As numerical estimation, we will take $g_{A}^{b s}=1.83 \times 10^{-3}$, $g_{L}^{A b b}=-g_{R}^{A b b}=g_{A}^{q}$.

In the $S M$, the $F C$ decay $Z \rightarrow \bar{b} s+b \bar{s}$ originates from one loop diagrams with branching ratio $\sim 3 \times 10^{-8}[75-78]$. For future linear collider $(I L C)$, the expected sensitivity to the branching ratios of rare $Z$ decays can be improved from $10^{-5}$ at the $L E P$ to $10^{-8}$ at the Giga $Z[79,80]$. The new physics effects might be detectable via $Z \rightarrow b s$ if it indeed affects this decay. A lot of theoretical studies involving the $F C$ decay $Z \rightarrow b s$ have been given within some popular models beyond the $S M$, where its branching ratio can be significantly enhanced [81-92].

Using the effective couplings $g_{L}^{Z b s}$ and $g_{R}^{Z b s}$ given by eq. (2.4), we can easily calculate the partial width $\Gamma(Z \rightarrow \bar{b} s+b \bar{s})$. The numerical results for the branching ratio $\operatorname{Br}(Z \rightarrow$ $\bar{b} s+b \bar{s})=\Gamma(Z \rightarrow \bar{b} s+b \bar{s}) / \Gamma_{\text {total }}$ are shown in figure 2 , in which we have taken the $S M$ input parameters as: $\alpha_{s}\left(m_{Z}\right)=0.118, S_{W}^{2}=0.231, \Gamma_{\text {total }}=2.4945 \mathrm{GeV}$, and $M_{Z}=$ 91.1875GeV [93]. If the light axigluon can explain the $t \bar{t} F B A$ and at the same time satisfy 


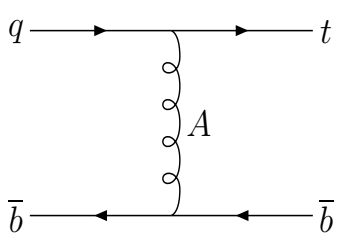

(a)

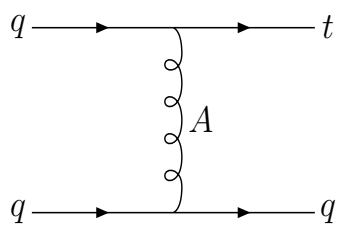

(d)

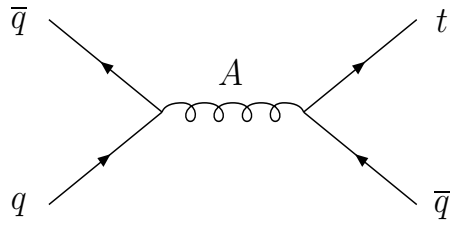

(b)

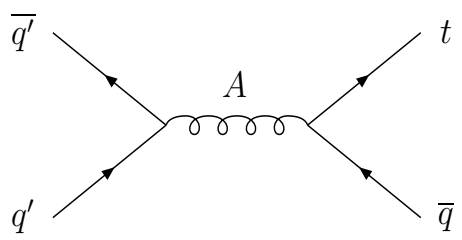

(e)

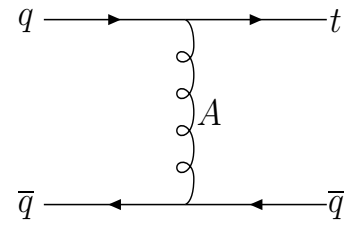

(c)

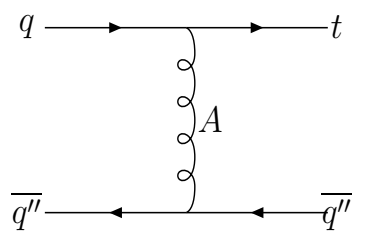

(f)

Figure 3. Leading order Feynman diagrams for $t \bar{b}$ and $t \bar{j}$ production contributed by the $F C$ couplings $g_{A}^{t q}$, in which $q=u, c, q^{\prime}=d, s, b$, and $q^{\prime \prime}=d, s$.

the constraints from the electroweak precision observables and the relevant data given by hadron colliders, its mass should be in the range of $100 \mathrm{GeV} \sim 400 \mathrm{GeV}$, its total decay width $\Gamma_{t}^{A}=(0.1 \sim 0.2) M_{A}$ and the flavor conserving coupling $g_{A}^{q}$ might be in the range of $0.3 \sim 0.5$ [33-39]. In our numerical estimation we have considered the effects of the axigluon width and taken $\Gamma_{t}^{A}=0.1 M_{A}$. For the mixing between the $S M$ and new quarks, we have taken case I and assumed $M_{H}=0.2 M_{A}$. One can see from figure 2 that, in most of the parameter space, the value of the branching ratio $\operatorname{Br}(Z \rightarrow \bar{b} s+b \bar{s})$ is smaller than $1 \times 10^{-8}$, which is still below the $S M$ prediction. So considering the constraints of $B_{s}^{0}-\overline{B_{s}^{0}}$ mixing on the $F C$ coupling $g_{A}^{b s}$, the contribution of the light axigluon to the rare decays $Z \rightarrow \bar{b} s$ and $b \bar{s}$ is very difficult to be detected in near future. Certainly, if we assume $\varepsilon_{H d} \neq V_{C K M}$, the numerical results should has some changes.

\section{The $F C$ couplings of the light axigluon $A$ and single top production at the $\mathrm{LHC}$}

In the $S M$, single top production dominantly occurs through electroweak processes, which are customary divided into three production channels: t-channel exchange of a space-like W boson, s-channel production and decay of a time-like $\mathrm{W}$ boson, and associated production of a top quark and an on-shell $\mathrm{W}$ boson. These partonic processes have their own distinct kinematics and do not interfcere with each other. Both at Tevatron and the $L H C$, the tchannel process is dominant one, which in five flavor $(5 F)$ scheme proceeds via the partonic processes $q b \rightarrow q^{\prime} t$ and $\bar{q} b \rightarrow \bar{q}^{\prime} t$ for single top production, and $q \bar{b} \rightarrow q^{\prime} \bar{t}$ and $\bar{q} \bar{b} \rightarrow \overline{q^{\prime}} \bar{t}$ for single antitop production. The s-channel partonic processes are $q \overline{q^{\prime}} \rightarrow t \bar{b}$ and $\bar{q} q^{\prime} \rightarrow \bar{t} b$ for single top and antitop productions, respectively. The contributions of charged and neutral color-octet vector bosons to top pairs and single top production has been studied in refs. [40, 94]. In this section we will consider the corrections of the light axigluon to the 
s- and t-channel single top productions via the $F C$ couplings $g_{A}^{t q}$ with $q=u$ or $c$. The relevant Feynman diagrams are shown in figure 3.

For the partonic process $q \bar{b} \rightarrow t \bar{b}$ as shown in figure 3 (a), the differential cross section with respect to emerging angle of the single top quark $\cos \theta_{t}$ can be written as

$$
\frac{d \sigma(t \bar{b})}{d \cos \theta_{t}}=\frac{2 \pi \alpha_{s}^{2} \beta\left(g_{A}^{t q}\right)^{2}\left(g_{A}^{b}\right)^{2}}{9 \hat{s}} P_{t}\left[\hat{s}\left(\hat{s}-m_{t}^{2}\right)+\hat{t}\left(\hat{t}-m_{t}^{2}\right)\right] .
$$

The partonic process $q \bar{q} \rightarrow t \bar{q}$ is composed of the s- and t-channel diagrams corresponding to figure 3 (b) and 3 (c). Its differential cross section is given by

$$
\begin{aligned}
\frac{d \sigma(t \bar{q})}{d \cos \theta_{t}}= & \frac{2 \pi \alpha_{s}^{2} \beta\left(g_{A}^{t q}\right)^{2}\left(g_{A}^{q}\right)^{2}}{9 \hat{s}}\left\{P_{s}\left[\hat{u}\left(\hat{u}-m_{t}^{2}\right)+\hat{t}\left(\hat{t}-m_{t}^{2}\right)\right]\right. \\
& -\frac{P_{s} P_{t}}{3}\left(\hat{s}-M_{A}^{2}\right)\left(\hat{t}-M_{A}^{2}\right) \hat{u}\left(\hat{u}-m_{t}^{2}\right) \\
& \left.+P_{t}\left[\hat{s}\left(\hat{s}-m_{t}^{2}\right)+\hat{u}\left(\hat{u}-m_{t}^{2}\right)\right]\right\}
\end{aligned}
$$

The differential cross section of the $\mathrm{t}+\mathrm{u}$ channel partonic process $q q \rightarrow t+q$ can be written as

$$
\begin{aligned}
\frac{d \sigma(t q)}{d \cos \theta_{t}}= & \frac{2 \pi \alpha_{s}^{2} \beta\left(g_{A}^{t q}\right)^{2}\left(g_{A}^{q}\right)^{2}}{9 \hat{s}}\left\{P_{t}\left[\hat{u}\left(\hat{u}-m_{t}^{2}\right)+\hat{s}\left(\hat{s}-m_{t}^{2}\right)\right]\right. \\
& +P_{t} P_{u}\left(\hat{t}-M_{A}^{2}\right)\left(\hat{u}-M_{A}^{2}\right) \hat{s}\left(\hat{s}-m_{t}^{2}\right) \\
& \left.+P_{u}\left[\hat{t}\left(\hat{t}-m_{t}^{2}\right)+\hat{s}\left(\hat{s}-m_{t}^{2}\right)\right]\right\} .
\end{aligned}
$$

The differential cross section for the s-channel partonic $\overline{q^{\prime}} q^{\prime} \rightarrow t \bar{q}$ as shown in figure 3 (e) is given by

$$
\frac{d \sigma_{s}(t \bar{q})}{d \cos \theta_{t}}=\frac{2 \pi \alpha_{s}^{2} \beta\left(g_{A}^{t q}\right)^{2}\left(g_{A}^{q^{\prime}}\right)^{2}}{9 \hat{s}} P_{s}\left[\hat{u}\left(\hat{u}-m_{t}^{2}\right)+\hat{t}\left(\hat{t}-m_{t}^{2}\right)\right]
$$

The explicit expression of the differential cross section for the t-channel $q \overline{q^{\prime \prime}} \rightarrow t \overline{q^{\prime \prime}}$ is same as that for the process $q \bar{b} \rightarrow t \bar{b}$, as long as replace the initial state $b$ quark by the quark $q^{\prime \prime}$ ( $d$ or $s$ ). In above equations, $\beta=1-\frac{m_{t}^{2}}{\hat{s}}, \hat{s}, \hat{t}$, and $\hat{u}$ are the usual Mandelstam variables,

$$
P_{i}=\frac{1}{\left(i-M_{A}^{2}\right)^{2}+M_{A}^{2} \Gamma_{A}^{2}} \quad \text { with } \quad i=\hat{s}, \hat{t}, \text { or } \hat{u} .
$$

Using above equations we can calculate the cross sections of $t b$ and $t j$ production at the $L H C$ induced by the light axigluon with the $F C$ coupling $g_{A}^{t q}$. In our numerical calculations, we use the leading order parton distribution function of CTEQ6L1 [95] and choose the factorization and renormalization scales to be $\mu_{f}=\mu_{r}=m_{t} / 2$ with $m_{t}=173 \mathrm{GeV}$. Our numerical results are added $t \bar{b}$ and $\bar{t} b$ for the process $p p \rightarrow t b$, and similar for $t j$ production with $j=u, c, d$, and $s$. It is obvious that the production cross sections depend on the mass parameter $M_{A}$, the coupling parameters $g_{A}^{t q}$ and $g_{A}^{q}$, where we have taken $g_{A}^{t u}=g_{A}^{t c}$ and the flavor conserving coupling $g_{A}^{q}$ being flavor universal.

In the $S M$, single top production at hadron colliders was first considered in ref. [96]. Now the production cross sections for the s- and t-channels have been calculated up to 


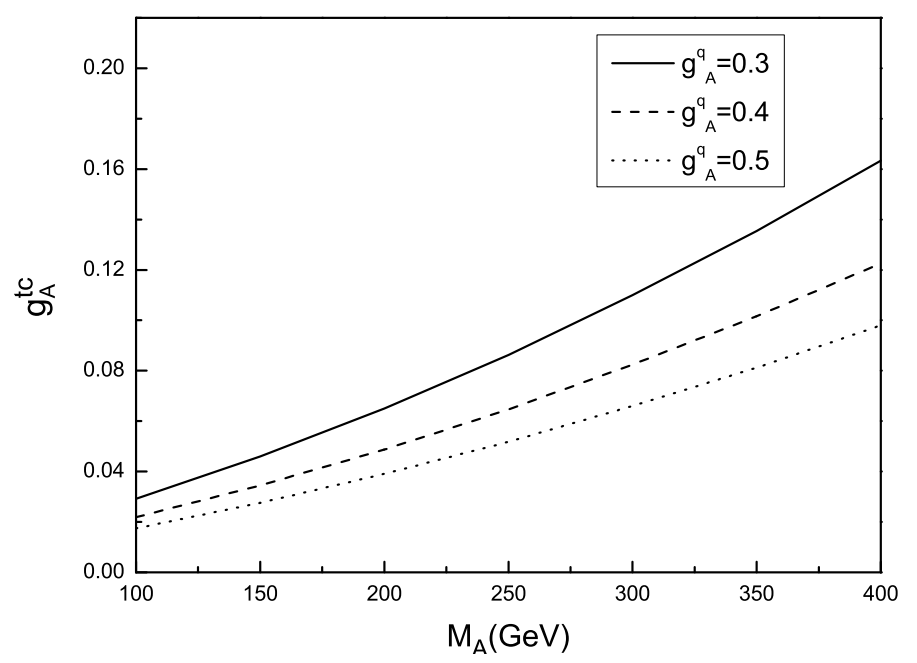

Figure 4. In the case of $\delta \sigma^{s} / \sigma_{S M}^{s}=10 \%$, the $F C$ coupling $g_{A}^{t q}$ as function of the axigluon mass $M_{A}$ for $g_{A}^{q}=0.3$ (solid line), 0.4 (dashed line) and 0.5(dotted line).

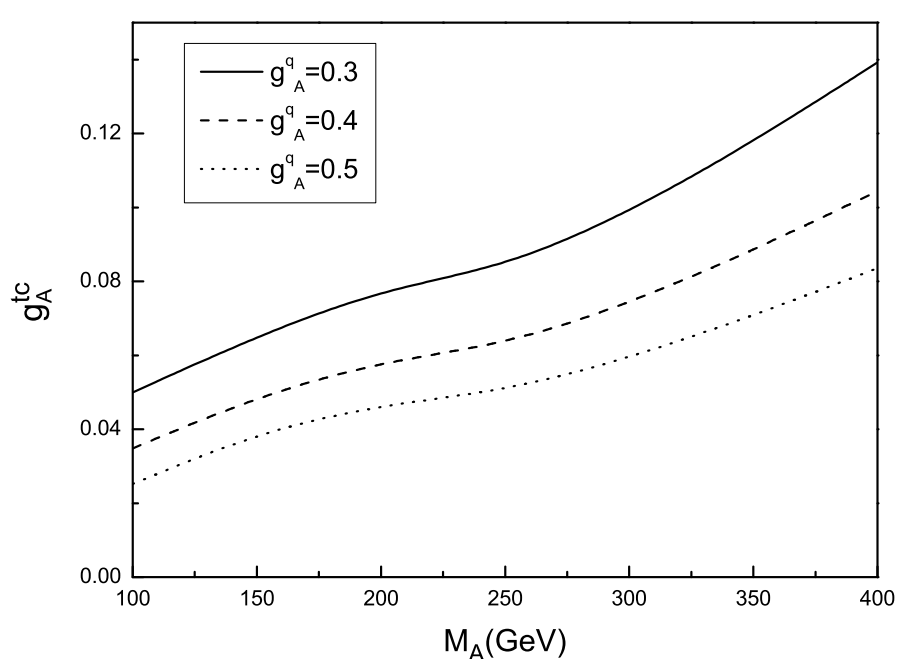

Figure 5. In the case of $\delta \sigma^{t} / \sigma_{S M}^{t}=10 \%$, the $F C$ coupling $g_{A}^{t q}$ as function of the axigluon mass $M_{A}$ for $g_{A}^{q}=0.3$ (solid line), 0.4 (dashed line) and 0.5(dotted line).

next-to-next-to leading logarithm $(N N L L)$ accuracy [97-102]: $\sigma_{s}=1.04 \pm 4 \% p b$ and $\sigma_{t}=2.26 \pm 5 \% p b$ at Tevatron with the centre-of-mass (c.m.) energy $\sqrt{s}=1.96 \mathrm{TeV}$ and $\sigma_{s}=12 \pm 6 \% p b$ and $\sigma_{t}=243 \pm 4 \% p b$ at the $L H C$ with $\sqrt{s}=14 \mathrm{TeV}$. The s- and t-channel cross sections have been measured at Tevatron by $C D F$ and $D O$ collaborations and the measurement precision can reach 18\% [57-61]. The measurement precision for the t-channel cross section at the $8 T e V L H C$ reported by $A T L A S$ and $C M S$ is about $15 \%$ [62-66]. It will be enhanced in coming years. For example, ref. [103] has shown that the cross section of the t-channel single top production at the $14 T e V L H C$ can be measured with a precision of $5 \%$. 


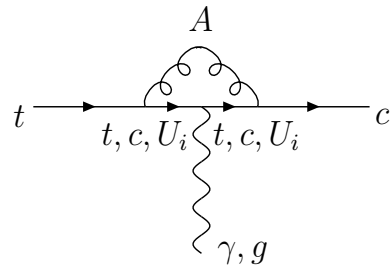

(a)

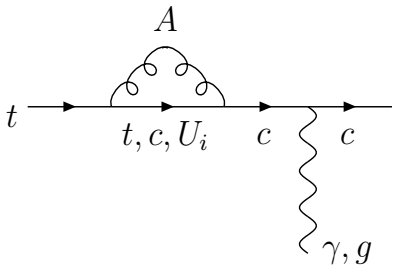

(b)

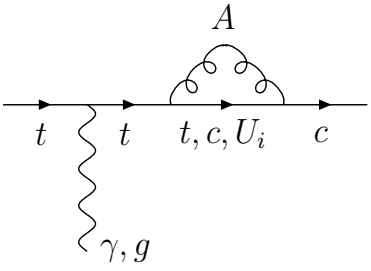

(c)

Figure 6. Feynman diagrams for the rare top decays $t \rightarrow c \gamma$ and $c g$ coming from the $F C$ coupling $g_{A}^{t c}$, in which $i=1$ and 2 .

From above discussions we can see that the theoretical error of the SM NNLO cross section at the $14 \mathrm{TeV} L H C$ for the s- and t-channel productions could be as large as $5 \%$, the same amount of the expected precision at the $14 \mathrm{TeV} \mathrm{LHC}$. So if the relative correction of the light axigluon to the single top production cross section is larger than $10 \%$, the $14 \mathrm{TeV} \mathrm{LHC}$ should detect this correction effect. In figure 4 and figure 5 we demand that $\delta \sigma^{s} / \sigma_{S M}^{s}=10 \%$ and $\delta \sigma^{t} / \sigma_{S M}^{t}=10 \%$, where $\sigma_{S M}^{s}$ and $\sigma_{S M}^{t}$ are the $S M N N L O$ predictions for the s- and t-channel single top production cross sections at the $L H C$ with $\sqrt{s}=14 T e V, \delta \sigma^{s}$ and $\delta \sigma^{t}$ are induced by the light axigluon $A$, and plot the $F C$ coupling $g_{A}^{t q}$ as a function of the mass parameter $M_{A}$ for different values of the flavor conserving $g_{A}^{q}$. In our numerical calculation, we have taken the central values for $\sigma_{S M}^{s}$ and $\sigma_{S M}^{t}$. From these figures one can see that the contributions of the light axigluon to the production cross sections of the processes $p p \rightarrow t b+X$ and $p p \rightarrow t j+X$ increase as the coupling parameters $g_{A}^{t q}$ and $g_{A}^{q}$ increasing, while decrease as $M_{A}$ increasing. For $100 \mathrm{GeV} \leq M_{A} \leq 400 \mathrm{GeV}$ and $0.3 \leq g_{A}^{q} \leq 0.5$, the values of $F C$ coupling $g_{A}^{t q}$ are in the ranges of $0.017 \sim 0.163$ and $0.024 \sim 0.139$ for $\delta \sigma^{s} / \sigma_{S M}^{s}=10 \%$ and $\delta \sigma^{t} / \sigma_{S M}^{t}=10 \%$, respectively. We expect that, in near future, the $L H C$ can authenticate this correction effect on single top production or at least give constraint on the $F C$ coupling $g_{A}^{t q}$.

\section{The light axigluon and the rare top decays $t \rightarrow c \gamma$ and $c g$}

It is well known that in the $S M$ the rare top decays $t \rightarrow q V(q=u, c$ and $V=\gamma, g, Z)$ mediated by FCNCs are highly GIM suppressed with branching ratios of $B r(t \rightarrow c V) \sim$ $10^{-14} \sim 10^{-12}[104,105]$, which are far below the detectable level of current or near future experiments. However, some new physics models can enhance these branching ratios significantly [106-108]. So rare top decays offer an opportunity to test the $S M$ and search for new physics effects. Any positive signal of rare top decay processes would clearly indicate new physics beyond the $S M$.

On the experimental side, rare top decays are being searched for at Tevatron $[109,110]$ and $L H C$ [111-113]. ATLAS collaboration has set upper limit on the branching ratio $\operatorname{Br}(t \rightarrow c g)<2.7 \times 10^{-4}$ at $95 \%$ C.L. [113]. The sensitivity of ATLAS to the branching ratio $B r(t \rightarrow c \gamma)$ is expected to be of the order of $10^{-4}$ [114]. 


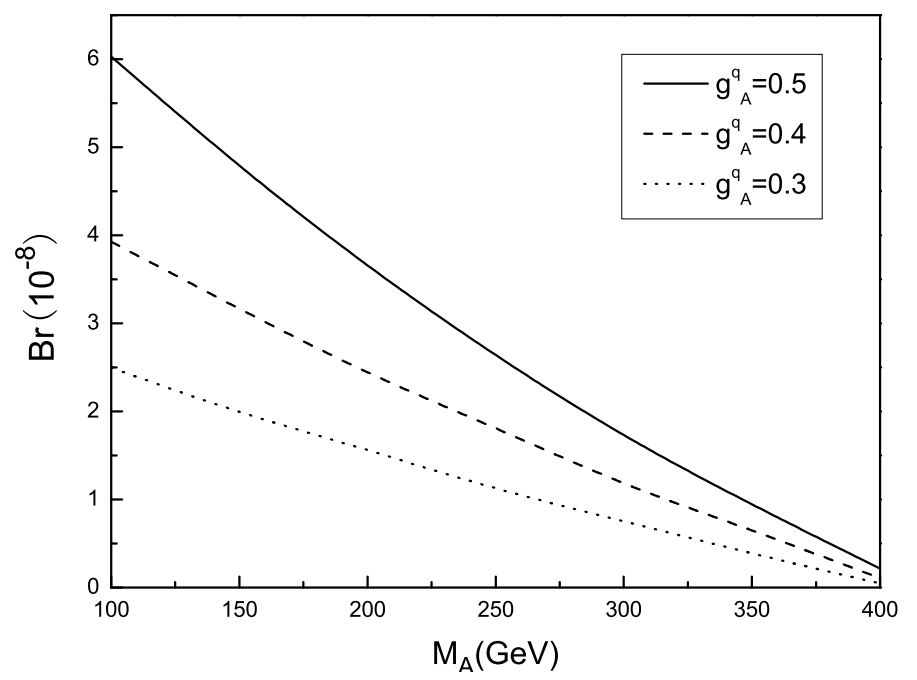

Figure 7. The branching ratio $B r(t \rightarrow c \gamma)$ as a function of the axigluon mass $M_{A}$ for three values of the flavor conserving coupling $g_{A}^{q}$.

From discussions given in above sections we can see that the light axigluon with $F C$ couplings can contribute rare top decays. In this section we will calculate the branching ratios $\mathrm{Br}(t \rightarrow c \gamma)$ and $\mathrm{Br}(t \rightarrow c g)$ induced by the light axigluon. The relevant Feynman diagrams are shown in figure 6 . In this section, we also assume that the contributions of the third generation new quarks to the rare top decays $t \rightarrow c \gamma$ and $t \rightarrow c g$ decouple. Compared to the $F C$ couplings of the light axigluon $A$ to the new quarks and the $S M$ quarks, the $F C$ couplings of the scalar $\phi$ to the new quarks and the $S M$ quarks arise at higher order, their $F C$ effects are much smaller than those induced by the axigluon $A$. Thus, in this section, we neglect the contributions of the scalar $\phi$ to the rare top decays $t \rightarrow c \gamma$ and $t \rightarrow c g$ as done for $Z \rightarrow b s$ in section 2 .

Considering electromagnetic gauge invariance, the amplitude of the rare decay $t \rightarrow c \gamma$ can be general written as

$$
M(t \rightarrow c \gamma)=i \bar{u}\left(P_{c}\right) \sigma^{\mu \nu} q_{\nu}\left(A_{\gamma}+B_{\gamma} \gamma_{5}\right) u\left(P_{t}\right) \varepsilon_{\mu}^{*}(q)
$$

where $q=P_{t}-P_{c}$ is the photon momentum and $\varepsilon$ is its polarization vector, in which $P_{t}$ and $P_{c}$ represent the momenta of top and charm quarks, respectively. A similar structure is valid for $t \rightarrow c g$ with form factors $A_{g}$ and $B_{g}$. For the light axigluon $A$ with zero vector couplings to the $S M$ and new quarks i.e. $g_{V}^{t q} \approx 0, g_{V}^{Q_{H} q} \approx 0$ and $g_{V}^{q} \approx 0[36,37,39]$, there are $A_{\gamma} \neq 0, A_{g} \neq 0$ and $B_{\gamma}=0, B_{g}=0$. Recently, ref. [115] has calculated the contributions of color-singlet gauge bosons predicted by the 331 models to the rare top decay $t \rightarrow c \gamma$ and give the explicit expressions for the relevant form factors. In this paper we will use LoopTools $[116,117]$ to obtain our numerical results.

Using eq. (4.1), the partial widths of $t \rightarrow c \gamma$ and $t \rightarrow c g$ contributed by the light 


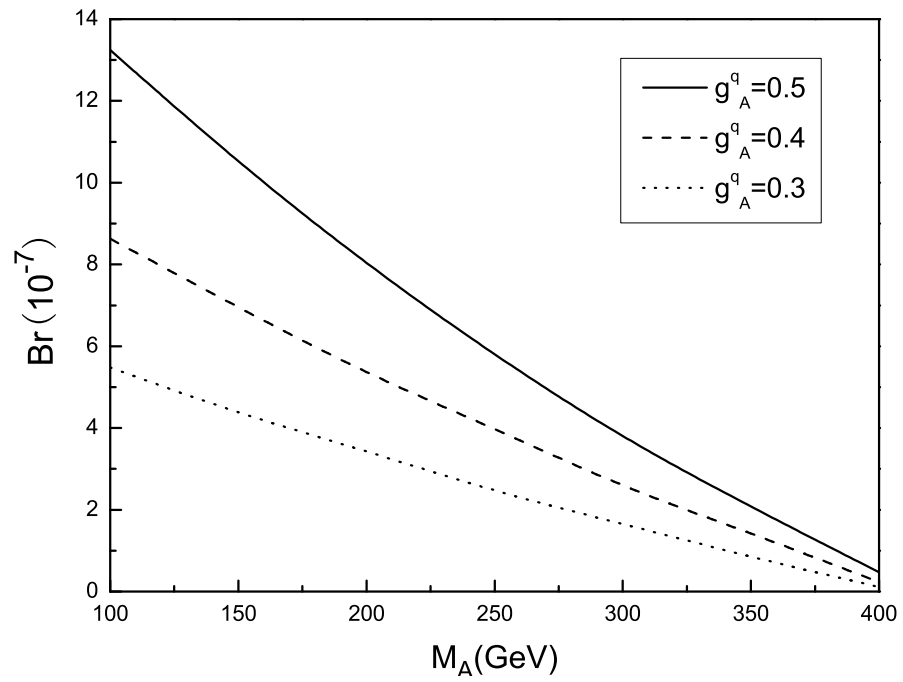

Figure 8. The branching ratio $B r(t \rightarrow c g)$ as a function of the axigluon mass $M_{A}$ for three values of the flavor conserving coupling $g_{A}^{q}$.

axigluon can be written as

$$
\begin{aligned}
\Gamma(t \rightarrow c \gamma) & =\frac{m_{t}^{3}}{8 \pi}\left(1-\frac{m_{c}^{2}}{m_{t}^{2}}\right)^{3}\left|A_{\gamma}\right|^{2}, \\
\Gamma(t \rightarrow c g) & =\frac{C_{F} m_{t}^{3}}{8 \pi}\left(1-\frac{m_{c}^{2}}{m_{t}^{2}}\right)^{3}\left|A_{g}\right|^{2},
\end{aligned}
$$

where $C_{F}=4 / 3$ is a color factor.

To obtain numerical results, we have assumed that the top total decay width is dominated by the decay $t \rightarrow W b$. The $F C$ coupling $g_{A}^{t c}$ is determined by the parameters $g_{A}^{q}$ and $M_{A}$ via the relation $\delta \sigma^{t} / \sigma_{S M}^{t}=10 \%$. For calculation the contributions of the first and second generation new quarks, we take the case II: $\varepsilon_{H d}=I, \quad \varepsilon_{H u}=V_{C K M}$ and assume $M_{H}=0.2 M_{A}$. In figure 7 and figure 8 we plot the branching ratios $B r(t \rightarrow c \gamma)$ and $B r(t \rightarrow c g)$ as functions of the axigluon mass $M_{A}$ for three values of the flavor conserving coupling $g_{A}^{q}$. One can see from these figures that the light axigluon $A$ can indeed enhance the branching ratios $B r(t \rightarrow c \gamma)$ and $B r(t \rightarrow c g)$. For $0.3 \leq g_{A}^{q} \leq 0.5$ and $100 \mathrm{GeV} \leq M_{A} \leq 400 \mathrm{GeV}$, the values of $\mathrm{Br}(t \rightarrow c \gamma)$ and $\mathrm{Br}(t \rightarrow c g)$ are in the ranges of $4.8 \times 10^{-9} \sim 5.9 \times 10^{-8}$ and $1.1 \times 10^{-8} \sim 1.3 \times 10^{-6}$, respectively. Replacing the $F C$ couplings $g_{A}^{t c}$ and $g_{A}^{U_{i} c}$ by $g_{A}^{t u}$ and $g_{A}^{U_{i} u}$, we can easily calculate the contributions of the light axigluon $A$ to the rare top decays $t \rightarrow u \gamma$ and $u g$.

\section{Conclusions}

The light axigluon $A$ with a mass $M_{A}$ in the range from $100 \mathrm{GeV}$ to $400 \mathrm{GeV}$ predicted by the light axigluon model $[36,37]$ can explain the $t \bar{t} F B A$ and satisfy the constraints from the $A T L A S$ and $C M S$ data, as long as its decay width is large and its couplings to the $S M$ quarks are relatively small. In order to get suppressed couplings of the light axigluon $A$ to 
the $S M$ quarks, the new quarks and the $S M$ quarks should have mixing, which can induce the $F C$ couplings to the new quarks and the $S M$ quarks. Furthermore, to fulfill the broad width of the axigluon, the new quarks, at least the first and second generation new quarks, are lighter than the light axigluon. In this paper, we assume the flavor conserving axigluon couplings are universal and pure axial vector-like, and investigate some $F C$ phenomena mediated by the light axigluon.

The contributions of the light axigluon model to the $F C$ decays $Z \rightarrow \bar{b} s, b \bar{s}$ and $t \rightarrow$ $c \gamma, c g$ mainly come from the $F C$ quark- quark- axigluon coupling $g_{A}^{q q^{\prime}}$ and the $F C$ quarknew quark- axigluon coupling $g_{A}^{q Q_{H}}$. Considering the constraints of meson mixing on the $F C$ coupling $g_{A}^{q q^{\prime}}$ and assuming that both $\varepsilon_{H u}$ and $\varepsilon_{H d}$ are nearly equal to the identity matrices and satisfy the relation $\varepsilon_{H u}^{+} \varepsilon_{H d}=V_{C K M}$ to give the value of $g_{A}^{q Q_{H}}$, we calculate the branching ratios $\operatorname{Br}(Z \rightarrow \bar{b} s+b \bar{s}), B r(t \rightarrow c \gamma)$ and $B r(t \rightarrow c g)$ in the context of the light axigluon model. Our numerical results show that, in most of parameter space, the value of the branching ratio $\operatorname{Br}(Z \rightarrow \bar{b} s+b \bar{s})$ is smaller than $1 \times 10^{-8}$, which is still below the $S M$ prediction. Compared to the $S M$ predictions, the branching ratios $B r(t \rightarrow c \gamma)$ and $B r(t \rightarrow c g)$ can be significantly enhanced in the light axigluon model, while are still lower than the corresponding current experimental upper limits.

It is well known that single top production is very sensitive to new physics beyond the $S M$, whose effects can be assessed by precise measurement of the production cross section. In this paper, we study the correction effects of the light axigluon $A$ to the s- and t-channel single top productions at the $L H C$. We find that, in near future, the $L H C$ should observe this correction effect with reasonable values for the $F C$ coupling $g_{A}^{t q}$ or at least give constraint on the $F C$ coupling $g_{A}^{t q}$. If one demands $\delta \sigma^{s} / \sigma_{S M}^{s}=10 \%$ and

$\delta \sigma^{t} / \sigma_{S M}^{t}=10 \%$, the values of the $F C$ coupling $g_{A}^{t q}$ should be in the ranges of $0.017 \sim 0.163$ and $0.024 \sim 0.139$, respectively.

\section{Acknowledgments}

This work was supported in part by the National Natural Science Foundation of China under Grants No. 11275088 and Foundation of Liaoning Educational Committee (No. LT2011015).

Open Access. This article is distributed under the terms of the Creative Commons Attribution License (CC-BY 4.0), which permits any use, distribution and reproduction in any medium, provided the original author(s) and source are credited.

\section{References}

[1] ATLAS collaboration, Observation of a new particle in the search for the Standard Model Higgs boson with the ATLAS detector at the LHC, Phys. Lett. B 716 (2012) 1 [arXiv: 1207.7214] [INSPIRE].

[2] CMS collaboration, Observation of a new boson at a mass of $125 \mathrm{GeV}$ with the CMS experiment at the LHC, Phys. Lett. B 716 (2012) 30 [arXiv:1207.7235] [INSPIRE]. 
[3] C.T. Hill, Topcolor: Top quark condensation in a gauge extension of the standard model, Phys. Lett. B 266 (1991) 419 [INSPIRE].

[4] C.T. Hill, Topcolor assisted technicolor, Phys. Lett. B 345 (1995) 483 [hep-ph/9411426] [INSPIRE].

[5] R.S. Chivukula, A.G. Cohen and E.H. Simmons, New strong interactions at the Tevatron?, Phys. Lett. B 380 (1996) 92 [hep-ph/9603311] [InSPIRE].

[6] E.H. Simmons, Coloron phenomenology, Phys. Rev. D 55 (1997) 1678 [hep-ph/9608269] [INSPIRE].

[7] J.C. Pati and A. Salam, Are the New Particles Color Gluons?, Phys. Rev. Lett. 34 (1975) 613 [INSPIRE].

[8] P.H. Frampton and S.L. Glashow, Chiral Color: An Alternative to the Standard Model, Phys. Lett. B 190 (1987) 157 [inSPIRE].

[9] P.H. Frampton and S.L. Glashow, Unifiable Chiral Color With Natural Gim Mechanism, Phys. Rev. Lett. 58 (1987) 2168 [INSPIRE].

[10] J. Bagger, C. Schmidt and S. King, Axigluon Production in Hadronic Collisions, Phys. Rev. D 37 (1988) 1188 [INSPIRE].

[11] R.S. Chivukula, E.H. Simmons and C.-P. Yuan, Axigluons cannot explain the observed top quark forward-backward asymmetry, Phys. Rev. D 82 (2010) 094009 [arXiv:1007.0260] [INSPIRE].

[12] P.H. Frampton, J. Shu and K. Wang, Axigluon as Possible Explanation for $p \bar{p} \rightarrow t \bar{t}$ Forward-Backward Asymmetry, Phys. Lett. B 683 (2010) 294 [arXiv:0911.2955] [INSPIRE].

[13] D.A. Dicus, C.D. McMullen and S. Nandi, Collider implications of Kaluza-Klein excitations of the gluons, Phys. Rev. D 65 (2002) 076007 [hep-ph/0012259] [INSPIRE].

[14] E. Farhi and L. Susskind, Technicolor, Phys. Rept. 74 (1981) 277 [InSPIRE].

[15] K. Lane and S. Mrenna, The Collider phenomenology of technihadrons in the technicolor straw man model, Phys. Rev. D 67 (2003) 115011 [hep-ph/0210299] [INSPIRE].

[16] CDF collaboration, T. Aaltonen et al., Forward-Backward Asymmetry in Top Quark Production in $p \bar{p}$ Collisions at sqrts $=1.96$ TeV, Phys. Rev. Lett. 101 (2008) 202001 [arXiv: 0806.2472] [INSPIRE].

[17] CDF collaboration, T. Aaltonen et al., Evidence for a Mass Dependent Forward-Backward Asymmetry in Top Quark Pair Production, Phys. Rev. D 83 (2011) 112003 [arXiv:1101.0034] [INSPIRE].

[18] D0 collaboration, V.M. Abazov et al., First measurement of the forward-backward charge asymmetry in top quark pair production, Phys. Rev. Lett. 100 (2008) 142002 [arXiv: 0712.0851] [INSPIRE].

[19] P. Ferrario and G. Rodrigo, Massive color-octet bosons and the charge asymmetries of top quarks at hadron colliders, Phys. Rev. D 78 (2008) 094018 [arXiv:0809.3354] [INSPIRE].

[20] M.V. Martynov and A.D. Smirnov, Chiral color symmetry and possible G-prime-boson effects at the Tevatron and LHC, Mod. Phys. Lett. A 24 (2009) 1897 [arXiv:0906.4525] [INSPIRE]. 
[21] P. Ferrario and G. Rodrigo, Constraining heavy colored resonances from top-antitop quark events, Phys. Rev. D 80 (2009) 051701 [arXiv:0906.5541] [INSPIRE].

[22] Q.-H. Cao, D. McKeen, J.L. Rosner, G. Shaughnessy and C.E.M. Wagner, Forward-Backward Asymmetry of Top Quark Pair Production, Phys. Rev. D 81 (2010) 114004 [arXiv:1003.3461] [INSPIRE].

[23] R.S. Chivukula, E.H. Simmons and C.-P. Yuan, Axigluons cannot explain the observed top quark forward-backward asymmetry, Phys. Rev. D 82 (2010) 094009 [arXiv:1007.0260] [INSPIRE].

[24] Y. Bai, J.L. Hewett, J. Kaplan and T.G. Rizzo, LHC Predictions from a Tevatron Anomaly in the Top Quark Forward-Backward Asymmetry, JHEP 03 (2011) 003 [arXiv:1101.5203] [INSPIRE].

[25] A.R. Zerwekh, The Axigluon, a Four-Site Model and the Top Quark Forward-Backward Asymmetry at the Tevatron, Phys. Lett. B 704 (2011) 62 [arXiv:1103.0956] [INSPIRE].

[26] M.I. Gresham, I.-W. Kim and K.M. Zurek, On Models of New Physics for the Tevatron Top $A_{F B}$, Phys. Rev. D 83 (2011) 114027 [arXiv:1103.3501] [InSPIRE].

[27] A. Djouadi, G. Moreau and F. Richard, Forward-backward asymmetries of the bottom and top quarks in warped extra-dimensional models: LHC predictions from the LEP and Tevatron anomalies, Phys. Lett. B 701 (2011) 458 [arXiv:1105.3158] [INSPIRE].

[28] J. Cao, L. Wu and J.M. Yang, New physics effects on top quark spin correlation and polarization at the LHC: a comparative study in different models, Phys. Rev. D 83 (2011) 034024 [arXiv:1011.5564] [InSPIRE].

[29] E. Alvarez, L. Da Rold, J.I.S. Vietto and A. Szynkman, Phenomenology of a light gluon resonance in top-physics at Tevatron and LHC, JHEP 09 (2011) 007 [arXiv:1107.1473] [INSPIRE].

[30] J.A. Aguilar-Saavedra and M. Pérez-Victoria, Shaping the top asymmetry, Phys. Lett. B 705 (2011) 228 [arXiv:1107.2120] [InSPIRE].

[31] H. Wang, Y.-k. Wang, B. Xiao and S.-h. Zhu, New color-octet axial vector boson revisited, Phys. Rev. D 84 (2011) 094019 [arXiv:1107.5769] [INSPIRE].

[32] G.Z. Krnjaic, Very Light Axigluons and the Top Asymmetry, Phys. Rev. D 85 (2012) 014030 [arXiv:1109.0648] [InSPIRE].

[33] J. Drobnak, J.F. Kamenik and J. Zupan, Flipping t tbar Asymmetries at the Tevatron and the LHC, Phys. Rev. D 86 (2012) 054022 [arXiv: 1205.4721] [InSPIRE].

[34] M. Cvetič, J. Halverson and P. Langacker, Ultraviolet Completions of Axigluon Models and Their Phenomenological Consequences, JHEP 11 (2012) 064 [arXiv: 1209.2741] [INSPIRE].

[35] B. Daz and A.R. Zerwekh, Axigluon Phenomenology using ATLAS dijet data, Int. J. Mod. Phys. A 28 (2013) 1350133 [arXiv:1308.0166].

[36] G. Marques Tavares and M. Schmaltz, Explaining the t-tbar asymmetry with a light axigluon, Phys. Rev. D 84 (2011) 054008 [arXiv:1107.0978] [INSPIRE].

[37] C. Gross, G. Marques Tavares, M. Schmaltz and C. Spethmann, Light axigluon explanation of the Tevatron ttbar asymmetry and multijet signals at the LHC, Phys. Rev. D 87 (2013) 014004 [arXiv:1209.6375] [INSPIRE]. 
[38] U. Haisch and S. Westhoff, Massive Color-Octet Bosons: Bounds on Effects in Top-Quark Pair Production, JHEP 08 (2011) 088 [arXiv: 1106.0529] [INSPIRE].

[39] M. Gresham, J. Shelton and K.M. Zurek, Open windows for a light axigluon explanation of the top forward-backward asymmetry, JHEP 03 (2013) 008 [arXiv: 1212.1718] [INSPIRE].

[40] S. Dutta, A. Goyal and M. Kumar, Top quark physics in the vector color-octet model, Phys. Rev. D 87 (2013) 094016 [arXiv:1209.3636] [INSPIRE].

[41] ATLAS collaboration, Search for New Physics in Dijet Mass and Angular Distributions in pp Collisions at $\sqrt{s}=7 \mathrm{TeV}$ Measured with the ATLAS Detector,

New J. Phys. 13 (2011) 053044 [arXiv:1103.3864] [inSPIRE].

[42] ATLAS collaboration, Search for Massive Colored Scalars in Four-Jet Final States in $\sqrt{s}=7$ TeV proton-proton collisions with the ATLAS Detector,

Eur. Phys. J. C 71 (2011) 1828 [arXiv:1110.2693] [INSPIRE].

[43] ATLAS collaboration, Search for New Physics in the Dijet Mass Distribution using $1 \mathrm{fb}^{-1}$ of pp Collision Data at $\sqrt{s}=7 \mathrm{TeV}$ collected by the ATLAS Detector,

Phys. Lett. B 708 (2012) 37 [arXiv:1108.6311] [INSPIRE].

[44] ATLAS collaboration, Search for top-jet resonances in the lepton+jets channel of ttbar + jets events with the ATLAS detector in $4.7 \mathrm{fb}-1$ of $\mathrm{pp}$ collisions at $\sqrt{s}=7 \mathrm{TeV}$, ATLAS-CONF-2012-096 (2012).

[45] ATLAS collaboration, Search for Massive Coloured Scalars with the ATLAS Detector in Four-Jet Final States using $4.6 \mathrm{fb}-1$ of $\sqrt{s}=7 \mathrm{TeV}$ proton-proton collision data, ATLAS-CONF-2012-110 (2012).

[46] CMS collaboration, Search for Dijet Resonances in $7 \mathrm{TeV}$ pp Collisions at CMS, Phys. Rev. Lett. 105 (2010) 211801 [arXiv:1010.0203] [INSPIRE].

[47] CMS collaboration, Search for Resonances in the Dijet Mass Spectrum from $7 \mathrm{TeV} \mathrm{pp}$ Collisions at CMS, Phys. Lett. B 704 (2011) 123 [arXiv:1107.4771] [INSPIRE].

[48] CMS collaboration, Inclusive and differential measurements of the $t \bar{t}$ charge asymmetry in proton-proton collisions at 7 TeV, Phys. Lett. B 717 (2012) 129 [arXiv:1207.0065] [INSPIRE].

[49] CMS collaboration, Search for narrow resonances and quantum black holes in inclusive and b-tagged dijet mass spectra from pp collisions at $\sqrt{s}=7$ TeV, JHEP 01 (2013) 013 [arXiv:1210.2387] [INSPIRE].

[50] CMS collaboration, Search for narrow resonances using the dijet mass spectrum in pp collisions at $\sqrt{s}=8 \mathrm{TeV}$, Phys. Rev. D 87 (2013) 114015 [arXiv:1302.4794] [INSPIRE].

[51] W. Bernreuther, Top quark physics at the LHC, J. Phys. G 35 (2008) 083001 [arXiv:0805.1333] [INSPIRE].

[52] J.R. Incandela, A. Quadt, W. Wagner and D. Wicke, Status and Prospects of Top-Quark Physics, Prog. Part. Nucl. Phys. 63 (2009) 239 [arXiv:0904.2499] [INSPIRE].

[53] F.P. Schilling, Top Quark Physics at the LHC: A Review of the First Two Years, Int. J. Mod. Phys. A 27 (2012) 1230016 [arXiv:1308.0166].

[54] T.M.P. Tait and C.-P. Yuan, Single top quark production as a window to physics beyond the standard model, Phys. Rev. D 63 (2000) 014018 [hep-ph/0007298] [INSPIRE]. 
[55] E. Boos and L. Dudko, The Single Top Quark Physics, Int. J. Mod. Phys. A 27 (2012) 1230026 [arXiv: 1211.7146].

[56] P. Falgari, SM single-top production at hadron colliders, J. Phys. Conf. Ser. 452 (2013) 012016 [arXiv:1302.3699] [INSPIRE].

[57] D0 collaboration, V.M. Abazov et al., Observation of Single Top Quark Production, Phys. Rev. Lett. 103 (2009) 092001 [arXiv:0903.0850] [inSPIRE].

[58] CDF collaboration, T. Aaltonen et al., First Observation of Electroweak Single Top Quark Production, Phys. Rev. Lett. 103 (2009) 092002 [arXiv: 0903.0885] [INSPIRE].

[59] D0 collaboration, V.M. Abazov et al., Model-independent measurement of t-channel single top quark production in p p collisions at $\sqrt{s}=1.96$ TeV, Phys. Lett. B 705 (2011) 313 [arXiv:1105.2788] [INSPIRE].

[60] D0 collaboration, V.M. Abazov et al., Measurements of single top quark production cross sections and $\left|V_{t b}\right|$ in p p collisions at $\sqrt{s}=1.96$ TeV, Phys. Rev. D 84 (2011) 112001 [arXiv:1108.3091] [INSPIRE].

[61] D0 collaboration, V.M. Abazov et al., Evidence for s-channel single top quark production in $p \bar{p}$ collisions at $\sqrt{s}=1.96$ TeV, Phys. Lett. B 726 (2013) 656 [arXiv:1307.0731] [INSPIRE].

[62] ATLAS collaboration, Measurement of the t-channel single top-quark production cross section in pp collisions at $\sqrt{s}=7 \mathrm{TeV}$ with the ATLAS detector, Phys. Lett. B 717 (2012) 330 [arXiv:1205.3130] [INSPIRE].

[63] ATLAS collaboration, Measurement of t-Channel Single Top-Quark Production in pp Collisions at $\sqrt{s}=8 \mathrm{TeV}$ with the ATLAS detector, ATLAS-CONF-2012-132 (2012).

[64] CMS collaboration, Measurement of the t-channel single top quark production cross section in pp collisions at $\sqrt{s}=7$ TeV, Phys. Rev. Lett. 107 (2011) 091802 [arXiv:1106. 3052] [INSPIRE].

[65] CMS collaboration, Measurement of the single-top-quark t-channel cross section in pp collisions at $\sqrt{s}=7 \mathrm{TeV}$, JHEP 12 (2012) 035 [arXiv:1209.4533] [INSPIRE].

[66] CMS collaboration, Measurement of the single-top t-channel cross section in pp collisions at centre-of-mass energy of $8 \mathrm{TeV}$, CMS-PAS-TOP-12-011.

[67] ATLAS collaboration, Evidence for the associated production of a $W$ boson and a top quark in ATLAS at $\sqrt{s}=7 \mathrm{TeV}$, Phys. Lett. B 716 (2012) 142 [arXiv: 1205.5764] [INSPIRE].

[68] CMS collaboration, Evidence for associated production of a single top quark and $W$ boson in pp collisions at $\sqrt{s}=7 \mathrm{TeV}$, Phys. Rev. Lett. 110 (2013) 022003 [arXiv:1209.3489] [INSPIRE].

[69] S. Ipek, Light Axigluon Contributions to b $\bar{b}$ and c $c \bar{c}$ Asymmetry and Constraints on Flavor Changing Axigluon Currents, Phys. Rev. D 87 (2013) 116010 [arXiv:1301.3990] [INSPIRE].

[70] B.A. Dobrescu, K. Kong and R. Mahbubani, Massive color-octet bosons and pairs of resonances at hadron colliders, Phys. Lett. B 670 (2008) 119 [arXiv:0709.2378] [INSPIRE].

[71] M. Cvetič, J. Halverson and P. Langacker, Ultraviolet Completions of Axigluon Models and Their Phenomenological Consequences, JHEP 11 (2012) 064 [arXiv:1209.2741] [INSPIRE]. 
[72] J. Hubisz, S.J. Lee and G. Paz, The Flavor of a little Higgs with T-parity, JHEP 06 (2006) 041 [hep-ph/0512169] [INSPIRE].

[73] B. Holdom, New third family flavor physics: Vertex corrections, Phys. Lett. B 351 (1995) 279 [hep-ph/9502273] [INSPIRE].

[74] C.T. Hill and X.-m. Zhang, $Z \rightarrow b \bar{b}$ versus dynamical electroweak symmetry breaking involving the top quark, Phys. Rev. D 51 (1995) 3563 [hep-ph/9409315] [INSPIRE].

[75] M. Clements et al., Flavor-changing decays of the $Z^{0}$, Phys. Rev. D 27 (1983) 570 [INSPIRE].

[76] V. Ganapathy et al., Flavor-changing $Z$ decays: A window to ultraheavy quarks?, Phys. Rev. D 27 (1983) 579 [inSPIRE].

[77] W.-S. Hou, N.G. Deshpande, G. Eilam and A. Soni, CP Nonconservation in Decays of $W$ and $Z$ Bosons, Phys. Rev. Lett. 57 (1986) 1406 [inSPIRE].

[78] J. Bernabeu, M.B. Gavela and A. Santamaria, CP Violation at the $Z^{0}$ Peak, Phys. Rev. Lett. 57 (1986) 1514 [INSPIRE].

[79] ECFA/DESY LC Physics Working Group, J.A. Aguilar-Saavedra et al., TESLA: The Superconducting electron positron linear collider with an integrated $x$-ray laser laboratory. Technical design report. Part 3. Physics at an $e^{+} e^{-}$linear collider, hep-ph/0106315 [INSPIRE].

[80] H. Baer et al., The International Linear Collider Technical Design Report - Volume 2: Physics, arXiv:1306.6352 [INSPIRE].

[81] M.J. Duncan, Flavor Changing Decays of the $Z^{0}$ and Supersymmetry, Phys. Rev. D 31 (1985) 1139 [INSPIRE].

[82] F. Gabbiani, J.H. Kim and A. Masiero, $Z^{0} \rightarrow b \bar{s}$ and $Z^{0} \rightarrow \tau \bar{\mu}$ in SUSY: Are They Observable?, Phys. Lett. B 214 (1988) 398 [INSPIRE].

[83] C. Busch, Charged Higgs Bosons and Flavor Changing Z Decays, Nucl. Phys. B 319 (1989) 15 [InSPIRE].

[84] B. Mukhopadhyaya and A. Raychaudhuri, Can Flavor Changing Z Decay Provide a Test for Supersymmetry?, Phys. Rev. D 39 (1989) 280 [inSPIRE].

[85] W.-S. Hou and R.G. Stuart, Flavor Changing Decays of the $Z^{0}$ Boson to Massive Quarks in the Two Higgs Doublet Model, Phys. Lett. B 226 (1989) 122 [InSPIRE].

[86] B. Grzadkowski, J.F. Gunion and P. Krawczyk, Neutral current flavor changing decays for the $Z$ boson and the top quark in two Higgs doublet models, Phys. Lett. B 268 (1991) 106 [INSPIRE].

[87] X.-L. Wang, G.-R. Lu and Z.-J. Xiao, Flavor changing Z decay in the one generation technicolor models, Phys. Rev. D 51 (1995) 4992 [INSPIRE].

[88] D. Atwood, L. Reina and A. Soni, Phenomenology of two Higgs doublet models with flavor changing neutral currents, Phys. Rev. D 55 (1997) 3156 [hep-ph/9609279] [InSPIRE].

[89] D. Atwood, S. Bar-Shalom, G. Eilam and A. Soni, Flavor changing Z decays from scalar interactions at a giga $Z$ linear collider, Phys. Rev. D 66 (2002) 093005 [hep-ph/0203200] [INSPIRE].

[90] C.-x. Yue, H. Li and H.-j. Zong, Flavor changing $Z$ decay $Z \rightarrow b \bar{s}(\bar{b} s)$ in topcolor assisted technicolor models, Nucl. Phys. B 650 (2003) 290 [hep-ph/0211116] [INSPIRE]. 
[91] R. Mohanta, Implications of the non-universal $Z$ boson in FCNC mediated rare decays, Phys. Rev. D 71 (2005) 114013 [hep-ph/0503225] [INSPIRE].

[92] X.-F. Han, L. Wang and J.M. Yang, Higgs and Z-boson FCNC decays correlated with B-meson decays in littlest Higgs model with T-parity, Phys. Rev. D 78 (2008) 075017 [arXiv:0807.4480] [INSPIRE].

[93] Particle Data Group collaboration, J. Beringer et al., Review of Particle Physics (RPP), Phys. Rev. D 86 (2012) 010001 [inSPIRE].

[94] D. Karabacak, S. Nandi and S.K. Rai, Diquark resonance and single top production at the Large Hadron Collider, Phys. Rev. D 85 (2012) 075011 [arXiv:1201.2917] [InSPIRE].

[95] J. Pumplin et al., New generation of parton distributions with uncertainties from global QCD analysis, JHEP 07 (2002) 012 [hep-ph/0201195] [INSPIRE].

[96] S.S.D. Willenbrock and D.A. Dicus, Production of Heavy Quarks from W Gluon Fusion, Phys. Rev. D 34 (1986) 155 [inSPIRE].

[97] N. Kidonakis, Single top production at the Tevatron: Threshold resummation and finite-order soft gluon corrections, Phys. Rev. D 74 (2006) 114012 [hep-ph/0609287] [INSPIRE].

[98] N. Kidonakis, Higher-order soft gluon corrections in single top quark production at the LHC, Phys. Rev. D 75 (2007) 071501 [hep-ph/0701080] [INSPIRE].

[99] N. Kidonakis, Next-to-next-to-leading-order collinear and soft gluon corrections for t-channel single top quark production, Phys. Rev. D 83 (2011) 091503 [arXiv:1103.2792] [INSPIRE].

[100] N. Kidonakis, Differential and total cross sections for top pair and single top production, arXiv: 1205.3453 [INSPIRE].

[101] N. Kidonakis, NNLL threshold resummation for top-pair and single-top production, arXiv: 1210.7813 [INSPIRE].

[102] N. Kidonakis, Single top and top pair production, arXiv:1212.2844 [INSPIRE].

[103] B. Schoenrock, E. Drueke, B.A. Gonzalez and R. Schwienhorst, Single top quark cross section measurement in the t-channel at the high-luminosity LHC, arXiv:1308.6307 [INSPIRE].

[104] G. Eilam, J.L. Hewett and A. Soni, Rare decays of the top quark in the standard and two Higgs doublet models, Phys. Rev. D 44 (1991) 1473 [Erratum ibid. D 59 (1999) 039901] [INSPIRE].

[105] J.A. Aguilar-Saavedra, Top flavor-changing neutral interactions: Theoretical expectations and experimental detection, Acta Phys. Polon. B 35 (2004) 2695 [hep-ph/0409342] [INSPIRE].

[106] F. Larios, R. Martinez and M.A. Perez, New physics effects in the flavor-changing neutral couplings of the top quark, Int. J. Mod. Phys. A 21 (2006) 3473 [hep-ph/0605003] [INSPIRE].

[107] J.M. Yang, Probing New Physics from Top Quark FCNC Processes at LHC: A Mini Review, Int. J. Mod. Phys. A 23 (2008) 3343 [arXiv:0801.0210] [InSPIRE].

[108] J. Drobnak, Constraints on new physics from top quark decays at high precision, arXiv:1210.5051 [INSPIRE]. 
[109] CDF collaboration, T. Aaltonen et al., Search for the Flavor Changing Neutral Current Decay $t \rightarrow Z q$ in $p \bar{p}$ Collisions at $\sqrt{s}=1.96$ TeV, Phys. Rev. Lett. 101 (2008) 192002 [arXiv: 0805.2109] [INSPIRE].

[110] D0 collaboration, V.M. Abazov et al., Search for flavor changing neutral currents in decays of top quarks, Phys. Lett. B 701 (2011) 313 [arXiv:1103.4574] [INSPIRE].

[111] ATLAS collaboration, A search for flavour changing neutral currents in top-quark decays in pp collision data collected with the ATLAS detector at $\sqrt{s}=7$ TeV, JHEP 09 (2012) 139 [arXiv: 1206.0257] [INSPIRE].

[112] CMS collaboration, Search for flavor changing neutral currents in top quark decays in pp collisions at $7 \mathrm{TeV}$, Phys. Lett. B 718 (2013) 1252 [arXiv:1208.0957] [InSPIRE].

[113] ATLAS collaboration, Search for FCNC single top-quark production at $\sqrt{s}=7 \mathrm{TeV}$ with the ATLAS detector, Phys. Lett. B $\mathbf{7 1 2}$ (2012) 351 [arXiv:1203.0529] [INSPIRE].

[114] ATLAS collaboration, Study of ATLAS sensitivity to FCNC top decays, Eur. Phys. J. C 52 (2007) 999 [arXiv:0712.1127] [InSPIRE].

[115] I. Cortes-Maldonado, G. Hernandez-Tome and G. Tavares-Velasco, Decay $t \rightarrow c \gamma$ in models with SUL(3) $\times U X(1)$ gauge symmetry, Phys. Rev. D 88 (2013) 14011.

[116] T. Hahn and M. Pérez-Victoria, Automatized one loop calculations in four-dimensions and D-dimensions, Comput. Phys. Commun. 118 (1999) 153 [hep-ph/9807565] [INSPIRE].

[117] T. Hahn, New features in FormCalc 4, Nucl. Phys. Proc. Suppl. 135 (2004) 333 [hep-ph/0406288] [INSPIRE]. 